\title{
Three-dimensional sonographic assessment of placental volume and vascularization in pregnancies complicated by hypertensive disorders : Case control study
}

Original

Article

Abdelhamid Shaheen, Mohamd Emarh, Heba Magd, Hamed Yassin

Obstetrics and Gynecology Department, Menofiya University, Menofiya, Egypt

\begin{abstract}
Introduction: Pre-eclampsia remains a leading cause of maternal and fetal morbidity and mortality.

Aim of the work: To compare placental volumes and vascularization between normotensive pregnant women and women with hypertensive disorders using three-dimensional sonography.

Patients and Methods: The study comprised of 60 patients divided into three groups; control group included 20 women with normal blood pressure, 20 patients with chronic hypertension (those with a diagnosis of elevated blood pressure before or within the first half of pregnancy) and 20 patients with mild pre-eclampsia (those with no history of arterial hypertension before pregnancy and a confirmed diagnosis of arterial hypertension associated with proteinuria in second half of pregnancy).

Results: there was no statistical difference between the three groups as regarding the demographic data. There was statistical significant difference between the three groups as regarding PV to FWT ratio. The mean of PV to FWT ratio of group 1, group 2 and group 3 was $0.1347 \pm 0.1590,0.1257 \pm 0.01,0.1408 \pm 0.017$, respectively, with significant statistical differences between normotensive and chronic hypertensive groups $(P=0.016)$ and with significant statistical differences between normotensive and mild pre-eclamptic group $(P=0.002)$. As regarding placental vascular indices, there was significant statistical differences between the three groups and VFI $(P=0.03)$, but there was no significant statistical difference between the three groups as regarding placental FI $(P=143)$. There was significant positive correlation between placental volume, gestational age and observed to expected PV ratio (r) $0.515,0.797$ respectively. There was significant negative correlation between PV and placental VI. (r) 0.247.

Conclusion: Infertile women are susceptible to violence. All women attended infertility clinics must be screened for presence of violence and offered adequate support. Healthcare staff should consider husband attendance and good counseling about the drawback of all types of violence.
\end{abstract}

Key Words: Hypertensive disorders, placental volume, pre-eclampsia, three-dimensional ultrasound, vascular indices.

Received: 14 June 2018, Accepted: 27 June 2018

Corresponding Author: Abdel-Hamid Shaheen, Department of Obstetrics and Gynecology, Menofia University, Egypt, Tel.: 01090595395, E-mail: AbdelhamidShaheen@yahoo.com.

ISSN: 2090-7265, August 2018, Vol.8, No.3

\section{INTRODUCTION}

Hypertensive disorders in pregnancy are responsible for substantial maternal and fetal morbidity and mortality ${ }^{[1]}$. Amongthehypertensivedisorders, preeclampsia has proven to be difficult to predict. Preeclampsia is a systemic syndrome that is typically characterized by new onset hypertension and proteinuria (urinary excretion of $\geq 300 \mathrm{mg}$ of protein in 24hrs) in pregnancy ${ }^{[2]}$. Multiple tests have been proposed as screening tests for preeclampsia, including analysis of maternal serum biochemical markers and sonographic parameters ${ }^{[3]}$. Doppler ultrasound studies of the uteroplacental circulation in the second trimester have demonstrated that the increased impedance to flow in these vessels is associated with an increased risk for subsequent development of preeclampsia and fetal growth restriction $^{[4]}$. To date, results have been conflicting. With regard to the use of sonography, various parameters have been proposed for screening, including Doppler evaluation of the uterine arteries and 3-dimensional (3D) placental volumes $^{[5]}$.

\section{PATIENTS AND METHODS}

A case control trial included 60 patients among those attending the outpatient clinic of obstetrics and gynecology in Shebien El-Kom teatching hospital, Menoufia, Egypt in the period between January 2017-2018 after giving informed written consent.

A total sample size of 60 patients were included in the study, their age ranged between 22-45 years and they were pregnant in single viable intrauterine fetus, their gestational age ranged from 28 to 40 weeks. 
Patients with any additional comorbidity including maternal disease, smoking, drug use and known fetal malformation were excluded from the study.

The participants were divided into three groups: Group 1 included 20 women with normal blood pressure (control group). Group 2 included 20 women with chronic hypertension (those with a diagnosis of elevated blood pressure before or within the first half of pregnancy). Group 3: included 20 women with mild preeclampsia (those with no history of arterial hypertension before pregnancy and a confirmed diagnosis of arterial hypertension associated with proteinuria in second half of pregnancy).

Mild preeclampsia is defined as diastolic blood pressure $\geq 90 \mathrm{mmHg}$ measured on two occasions at least 6 hours apart, combined with proteinuria (two or more occurrences of protein on dipstick, $>300 \mathrm{mmHg}$ total protein in a 24-hour urine collection, or a protein creatinine ratio $>300 \mathrm{mg} / \mathrm{mmol})^{[6]}$.

All patients were matched by maternal age, gestational age, and parity at the time of sonography with control patients who were healthy and normotensive.

Pregnancy dating was established by the date of last menstruation in association with first trimester sonographic measurement if available.

All participants were subjected to:

1. Full history.

2. General, abdominal and pelvic examination.

3. Laboratory investigations.

4. Then, each patient from each group underwent 3D ultrasound examination of the placenta, and the following data was collected:

1- The placental volume: measured by rational technique with 3D Virtual Organ Computer-Aided Analysis (VOCAL) software (sonoview; GE Healthcare). Since placental volumes vary throughout pregnancy, the observed values were compared to the expected values at the gestational age at which each participant will evaluated ${ }^{[7]}$

2-The placental vascular indices: were analyzed by 3D power Doppler sonography, they did not vary during pregnancy ${ }^{[8]}$; therefore, these indices were not adjusted for gestational age. They included vascularization index (VI), flow index (FL) and vascularization flow index (VFI).

3- Statistical analysis: results were analyzed by SPSS version 20 (SPSS Inc., Chicago, IL, USA) and Epi Info 2000. Two types of statistics were done: descriptive as percentage (\%), mean and standard deviation (SD) or analytical as One-way Anova test for comparison between more than two groups having quantitative variables and with independent parametric data, Kruskal Wallis test for comparison between more than two groups having quantitative variables and with independent nonparametric data, Post Hoc test multivariate hypothesis test was used for multiple comparisons between subgroups of sample having quantitative variables, Pearson correlation used to study the correlation between normally distributed quantitative variables and Roc-curve (Receiver Operating Characteristics curve) it was a plot of the true positive rate against the false positive rate for different possible cut-off of diagnostic test or marker. $P$-value at 0.05 was used to determine the significance regarding: P-value $<0.05$ to be statistically insignificant, $P$-value $\leq 0.05$ to be statistically significant and $P$-value $\leq 0.001$ to be highly statistically significant

\section{RESULTS}

The study was carried out on 60 pregnant women recruited from antenatal outpatient clinic and obstetric departments of Shebin El-Kom Teaching Hospital from January 2017 to January 2018. Among which, 20 women with normal blood pressure (control group) Group[1], 20 women with chronic hypertension (those with a diagnosis of elevated blood pressure before or within the first half of pregnancy) Group [2] and 20 women with mild preeclampsia (those with no history of arterial hypertension before pregnancy and a confirmed diagnosis of arteria hypertension associated with proteinuria in second half of pregnancy) Group [3].

As regarding patient characteristics, no statistically significant differences between the studied groups as regarding age, parity, gestational age at sonography in weeks and fetal weight at sonography in grams. (table 1) 
Table 1: Comparison between the studied groups regarding personal data

\begin{tabular}{|c|c|c|c|c|c|c|}
\hline Data & Total $(\mathrm{N}=60)$ & $\begin{array}{l}\text { Normotensive } \\
\text { group }(\mathrm{N}=20)\end{array}$ & $\begin{array}{l}\text { Chronic hypertensive group } \\
\qquad(\mathrm{N}=20)\end{array}$ & $\begin{array}{l}\text { Mild PE group } \\
\qquad(\mathrm{N}=20)\end{array}$ & Test of sig. & $P$-value \\
\hline \multicolumn{7}{|l|}{ Age (years) } \\
\hline - Mean \pm SD & $26.70 \pm 4.1$ & $25.8 \pm 3.3$ & $27.1 \pm 3.6$ & $27.1 \pm 5.2$ & $\mathrm{~F}$ & 0.49 \\
\hline - range & $22-40$ & $22-33$ & $22-33$ & $22-40$ & 0.714 & NS \\
\hline - median & 26.5 & 25 & 28 & 27 & & \\
\hline \multicolumn{7}{|l|}{ Parity } \\
\hline - Mean \pm SD & $1.50 \pm 1.18$ & $1.55 \pm 1.23$ & $1.80 \pm 1.28$ & $1.15 \pm 1.18$ & $\mathrm{~K}$ & 0.253 \\
\hline - range & $0-4$ & $0-4$ & $0-4$ & $0-3$ & 2.74 & NS \\
\hline - median & 1 & 1.50 & 2 & 1 & & \\
\hline \multicolumn{7}{|c|}{ GA by U/S in weeks } \\
\hline - Mean \pm SD & $35.67 \pm 2.15$ & $35.80 \pm 1.79$ & $35.70 \pm 2.71$ & $35.50 \pm 1.933$ & $\mathrm{~F}$ & 0.90 \\
\hline - range & 30-39 & $33-39$ & $30-39$ & $33-39$ & 0.098 & NS \\
\hline - median & 36 & 36 & 36.5 & 35 & & \\
\hline \multicolumn{7}{|l|}{ Fetal weight } \\
\hline - Mean \pm SD & $2741 \pm 477.3$ & $2895 \pm 337.1$ & $2767.5 \pm 489.1$ & $2562.5 \pm 543.5$ & $\mathrm{~F}$ & \\
\hline - range & $1500-3500$ & $2100-3400$ & $1500-3300$ & $1900-3500$ & 2.60 & $\begin{array}{c}0.083 \\
\text { NS }\end{array}$ \\
\hline - median & 2800 & 2900 & 2850 & 2650 & & \\
\hline
\end{tabular}

$\mathrm{F}=$ Anova test, $\mathrm{K}=$ Kruskal Wall test, $\mathrm{NS}=$ Non-significant

There were no statistically significant differences between the studied groups as regarding the mean of placental volume while there was significant difference as regarding $\mathrm{PV}$ to $\mathrm{FWT}$ ratio between normotensive
$(0.1347 \pm 0.1590)$ and chronic hypertensive groups $(0.1257$ $\pm 0.01)[P 1=0.016]$ and between normotensive $(0.1347 \pm$ $0.1590)$ and mild preeclamptic group $(0.1408 \pm 0.017)$ (table 2)

Table 2: comparison between the studied groups regarding PV and PV to FWT ratio

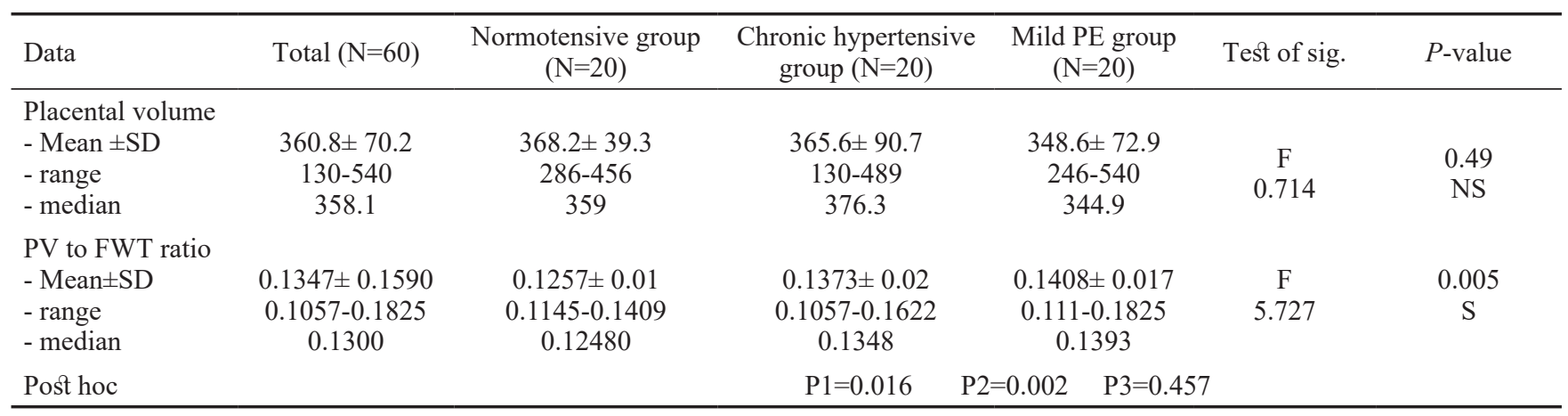

As regarding placental vascular indices, there were no statistically significant differences between the studied groups and the mean observe to expected PV ratio $(P=0.508)$ and the mean of placental flow index $(P=0.143)$ ; while there were highly significant statistically differences between the studied groups and the mean of placental vascularization index $(\mathrm{VI}=11.62 \pm 5.95)(P=0.001)$ with significant statistical differences between normotensive

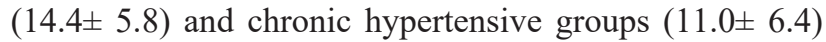
$(P 1=0.05)$ and with significant statistical differences between normotensive $(14.4 \pm 5.8)$ and mild preeclamptic group $(9.39 \pm 4.48) \quad(P 2=0.006)$ and non-significant statistical differences between chronic hypertensive and mild preeclamptic group $(P 3=0.374)$. Also, there were significant statistically differences between the studied groups and the mean of placental vascularization flow index $(\mathrm{VFI}=5.84 \pm 3.68)(P=0.01)$ with significant statistical differences between normotensive $(7.66 \pm 4.14)$ and chronic hypertensive groups $(5.29 \pm 3.69)(P 1=0.05)$ and with significant statistical differences between between normotensive $(7.66 \pm 4.14)$ and mild preeclamptic group $(4.58 \pm 2.40) \quad(P 2=0.007)$ and non-significant statistical differences between chronic hypertensive and mild preeclamptic group $(P 3=0.526)$ (table 3$)$ 
Table 3: Comparison between the studied groups regarding placental vascular indices

\begin{tabular}{|c|c|c|c|c|c|c|c|}
\hline Data & Total $(\mathrm{N}=60)$ & $\begin{array}{l}\text { Normotensive } \\
\text { group }(\mathrm{N}=20)\end{array}$ & $\begin{array}{l}\text { Chronic hypertensive } \\
\text { group }(\mathrm{N}=20)\end{array}$ & $\begin{array}{l}\text { Mild PE } \\
\text { group } \\
(\mathrm{N}=20)\end{array}$ & $\begin{array}{l}\text { Test } \\
\text { of sig }\end{array}$ & P-value & Post Hoc \\
\hline \multicolumn{8}{|l|}{ Observe to expected PV ratio } \\
\hline - Mean \pm SD & $0.9732 \pm 0.127$ & $0.964 \pm 0.06$ & $1.00 \pm 0.145$ & $0.955 \pm 0.151$ & $\mathrm{~F}$ & 0.508 & \\
\hline - range & $0.720-1.33$ & $0.836-1.131$ & $0.720-1.21$ & $0.777-1.33$ & 0.686 & NS & \\
\hline - median & 0.951 & 0.951 & 1 & 0.982 & & & \\
\hline \multicolumn{8}{|l|}{ Placental VI } \\
\hline - Mean \pm SD & $11.62 \pm 5.95$ & $14.4 \pm 5.8$ & $11.0 \pm 6.4$ & $9.39 \pm 4.48$ & $\mathrm{~K}$ & 0.001 & $\begin{array}{l}\mathrm{Pl}=0.05 \\
\mathrm{P} 2=0.006\end{array}$ \\
\hline - range & $1.91-34.2$ & $7.62-34.2$ & $6.44-34.1$ & $1.91-24.2$ & 13.9 & $\mathrm{~S}$ & $\begin{array}{l}\mathrm{P} 2=0.006 \\
\mathrm{P} 3=0374\end{array}$ \\
\hline - median & 10.2 & 12.5 & 9.12 & 9.14 & & & \\
\hline \multicolumn{8}{|l|}{ Placental FI } \\
\hline - Mean \pm SD & $43.2 \pm 2.89$ & $43.6 \pm 3.4$ & $43.83 \pm 2.5$ & $42.23 \pm 2.4$ & $\mathrm{~F}$ & 0.143 & \\
\hline - range & $36.1-50.9$ & $36.1-49.04$ & $40.3-50.9$ & $36.2-46.01$ & 2.01 & NS & \\
\hline - median & 43.1 & 43.7 & 43.1 & 42.1 & & & \\
\hline \multicolumn{8}{|l|}{ Placental VFI } \\
\hline - Mean \pm SD & $5.84 \pm 3.68$ & $7.66 \pm 4.14$ & $5.29 \pm 3.69$ & $4.58 \pm 2.40$ & $\begin{array}{c}0 \\
008\end{array}$ & 0.01 & $\begin{array}{c}P 4=0.03 \\
P 5=0.007\end{array}$ \\
\hline - range & $0.905-19.01$ & $2.90-19.01$ & $1.19-17.7$ & $0.905-12.3$ & 9.08 & $\mathrm{~S}$ & $\begin{array}{l}P 5=0.007 \\
P 6=0.526\end{array}$ \\
\hline - median & 5.1 & 7.1 & 4.7 & 4.21 & & & \\
\hline
\end{tabular}

There was no significant statistical correlation between placental volume and maternal age, parity, PV to FWT, placental FI and placental VFI. (r) 0.04, 0.06, 0.139, -0.07 and -0.07 , respectively. There was significant positive correlation between placental volume gestational age and observed to expected PV ratio. (r) 0.515, 0.797 respectively. There was significant negative correlation between PV and placental VI. (r) -0.247. (table 4, figure 1)

Table 4: Correlation between placental volume and other indices

\begin{tabular}{l|cc}
\hline & $\mathrm{R}$ & $P$-value \\
\hline Age & 0.04 & $0.73(\mathrm{NS})$ \\
Parity & 0.06 & $0.64(\mathrm{NS})$ \\
Gestational age/weeks & 0.515 & $0.001(\mathrm{~S})$ \\
PV to FWT ratio & 0.139 & $0.289(\mathrm{NS})$ \\
Obseve to expected PV ratio & 0.797 & $0.001(\mathrm{~S})$ \\
Placental VI & 0.247 & $0.05(\mathrm{~S})$ \\
Placental FI & 0.07 & $0.589(\mathrm{NS})$ \\
Placental VFI & 0.07 & $0.565(\mathrm{NS})$ \\
\hline
\end{tabular}
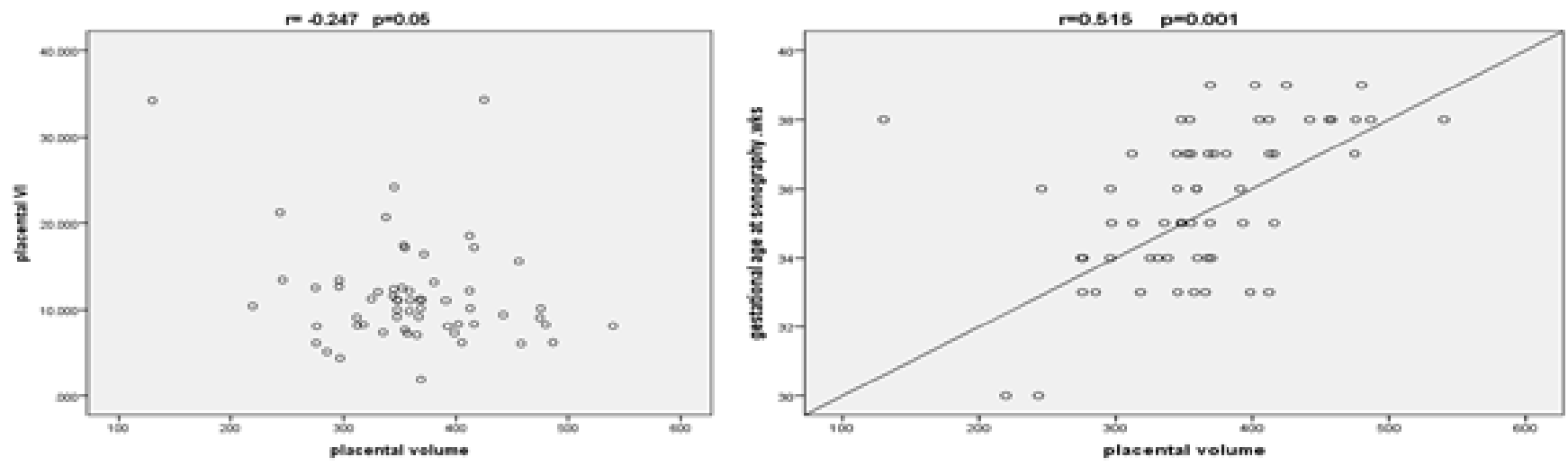

Fig. 1: Correlation between placental volume and other indices 
As regards the correlation between gestational age and placental volume and vascular indices, only there was significant positive correlation between gestational age and PV $(P=0.006)$ and placental VI $(P=0.01)$ in normotensive group, there was significant positive correlation between gestational age and PV $(P=0.04)$ and placental VFI $(P=0.01)$ in chronic hypertensive group and there was significant positive correlation between gestational age and PV $(P=0.006)$ only. (table 5 , figure 2 )

Table 5: Correlation between GA, PV and vascularization indices

\begin{tabular}{|c|c|c|c|c|c|c|}
\hline & \multicolumn{6}{|c|}{ Gestational age/week } \\
\hline & \multicolumn{2}{|c|}{ Normotensive group $(\mathrm{N}=20)$} & \multicolumn{2}{|c|}{ Chronic hypertensive group $(\mathrm{N}=20)$} & \multicolumn{2}{|c|}{ Mild PE group $(\mathrm{N}=20)$} \\
\hline & $\mathrm{r}$ & $P$-value & $\mathrm{R}$ & $P$-value & $\mathrm{r}$ & $P$-value \\
\hline Placental volume & 0.593 & $0.006(\mathrm{~S})$ & 0.459 & $0.04(\mathrm{~S})$ & 0.593 & $0.006(\mathrm{~S})$ \\
\hline Placental VI & 0.535 & $0.01(\mathrm{~S})$ & -0.108 & $0.65(\mathrm{NS})$ & 0.181 & $0.44(\mathrm{NS})$ \\
\hline Placental FI & -0.031 & $0.89(\mathrm{NS})$ & 0.35 & $0.88(\mathrm{NS})$ & 0.293 & $0.21(\mathrm{NS})$ \\
\hline Placental VFI & -0.399 & $0.08(\mathrm{NS})$ & 0.56 & $0.01(\mathrm{~S})$ & -0.15 & $0.51(\mathrm{NS})$ \\
\hline
\end{tabular}
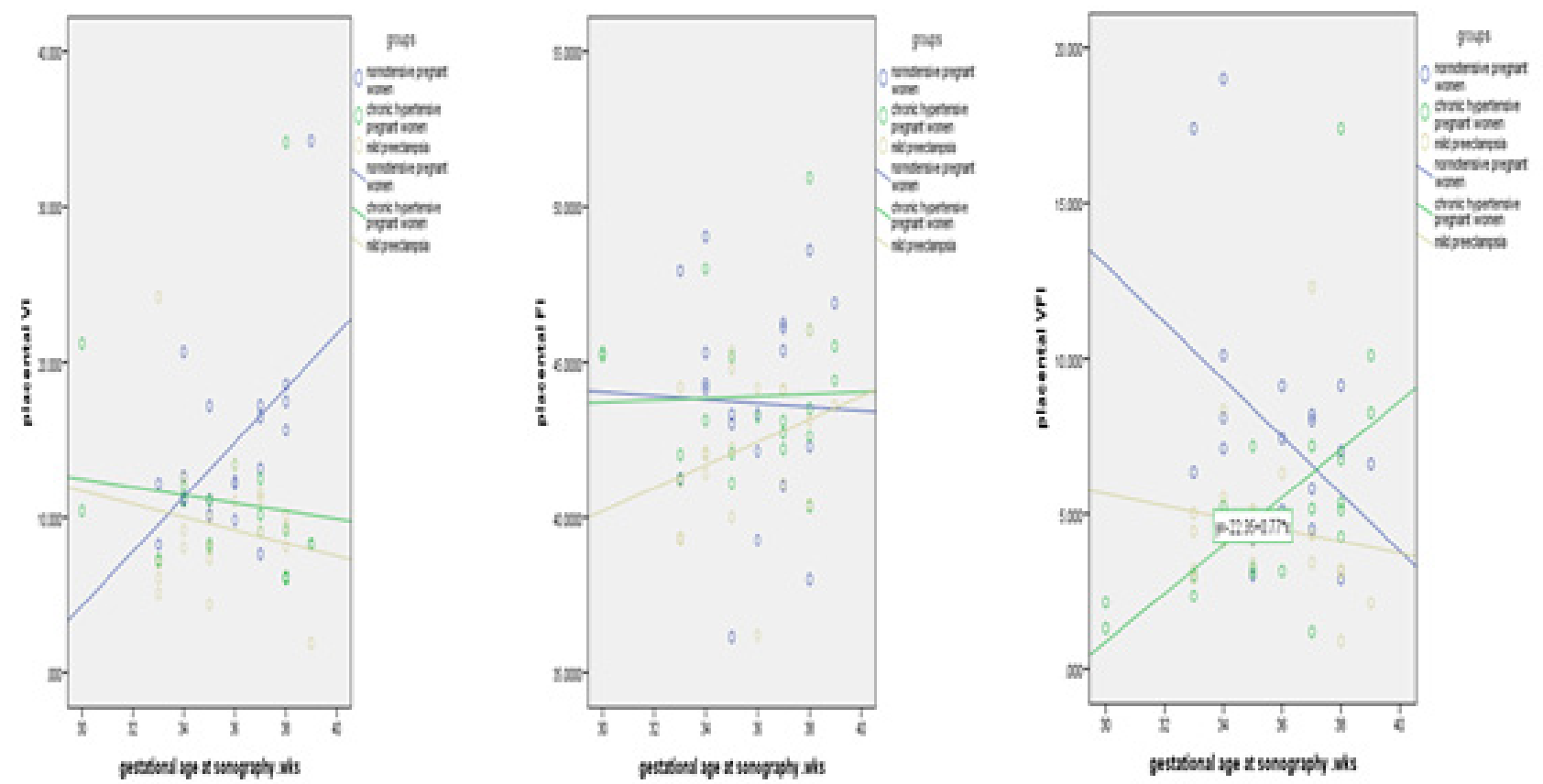

Fig. 2: ROC curve for each parameter.

For prediction of preeclampsia (table 6, figure 3):

- ROC curve shows placental volume at a cutoff $\geq 384.3$ can be used as a predictor of preeclampsia with sensitivity $60 \%$, specificity $70 \%$, negative predictive value $70 \%$ and positive predictive value $60 \%$.

- ROC curve shows placental VI at a cutoff $\geq 11.03$ can be used as a predictor of preeclampsia with sensitivity $75 \%$, specificity $57.5 \%$, negative predictive value $87.5 \%$ and positive predictive value $64.2 \%$.
- ROC curve shows placental FI at a cutoff \pm 44.1 can be used as a predictor of preeclampsia with sensitivity $75 \%$, specificity $55 \%$, negative predictive value $87.5 \%$ \& positive predictive value $68.7 \%$.

- ROC curve shows placental VFI at a cutoff $\geq 5.1$ can be used as a predictor of preeclampsia with sensitivity $80 \%$, specificity $57.5 \%$, negative predictive value $80 \%$ and positive predictive value $64.2 \%$. 
Table 6: Sensitivity and specificity placental volume, VI, FI, VFI in prediction of PE

\begin{tabular}{|c|c|c|c|c|c|c|}
\hline & AUC & Cutoff point & sensitivity & Specificity & NPV & PPV \\
\hline Placental volume & 0.635 & $\geq 384.3$ & $60 \%$ & $70 \%$ & $70 \%$ & $60 \%$ \\
\hline Placental VI & 0.686 & $\geq 11.03$ & $75 \%$ & $57.5 \%$ & $87.5 \%$ & $64.2 \%$ \\
\hline Placental FI & 0.654 & $\geq 44.1$ & $75 \%$ & $55 \%$ & $87.5 \%$ & $68.7 \%$ \\
\hline Placental VFI & 0.659 & $\geq 5.1$ & $80 \%$ & $57.5 \%$ & $80 \%$ & $64.2 \%$ \\
\hline
\end{tabular}
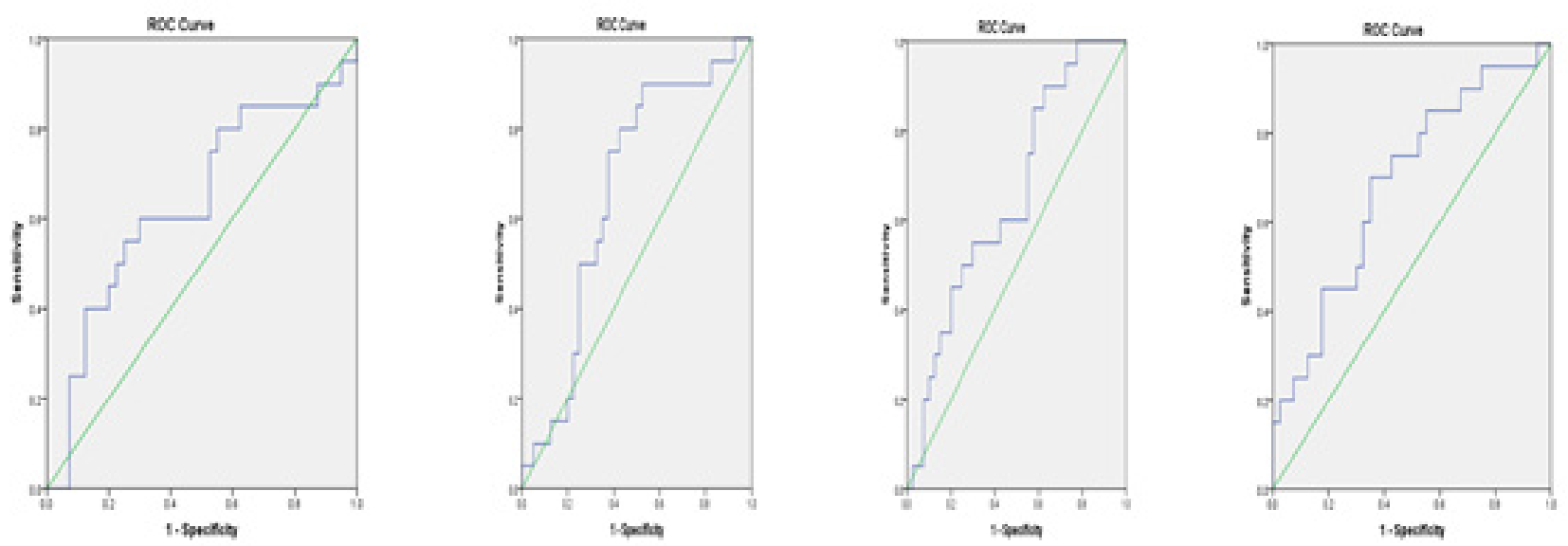

Fig. 3: Sensitivity and specificity placental volume, VI, FI, VFI in prediction of PE

\section{DISCUSSION}

This study was being conducted to evaluate the use of 3D ultrasonography in evaluation of placental volume and vascularization in pregnancies complicated by hypertensive disorders. In this study, placental volumes were assessed by 3D VOCAL software with rotation of 30 . As regarding maternal characteristics, there were no significant statistical differences between the different groups as well as shown in previous studies (most of them conducted at gestational age from 11 to $13+6 \mathrm{~d}$ weeks $)^{[7]}$. In this study, there was no significant statistical difference as regarding the placental volume $(P=0.508)$ and observed to expected placental volume ratio $(P=0.005)$ (as placental volumes vary throughout the gestational age) between normotensive and hypertensive groups. Also, there was significant statistical difference as regarding VI $(P=0.001)$ and VFI $(P=0.001)$ but not the placental FI $(P=0.143)$. These results were comparable to previous studies as shown in table 7 . 
Table 7: Study outcomes

\begin{tabular}{|c|c|c|c|c|c|c|c|}
\hline Study & Incidence of PE (\%) & Results/conclusion & \multicolumn{5}{|c|}{ Predictive measure } \\
\hline [9] & $\begin{array}{l}\text { - } 84 \text { PE }(8.5 \%) \\
-12 \text { early onset PE }(14.3 \%) \\
\text { - } 72 \text { late onset PE }(85.7 \%)\end{array}$ & $\begin{array}{c}\text { PE was associated with } \\
\text { significant lower PVI } \\
\cdot \text { VI: } P=0.008 \\
\text { - FI: } P<0.001 \\
\cdot \text { VFI: } P=0.004\end{array}$ & \multicolumn{5}{|c|}{ None } \\
\hline [10] & $\begin{array}{l}-20 \text { PE }(1.9 \%) \\
-4 \text { preterm PE }(0.4 \%) \\
-16 \text { term PE }(1.5 \%)\end{array}$ & $\begin{array}{l}\text { PE was associated with } \\
\text { significant lower PVI } \\
\text { • VI: } P<0.01 \\
\text { • FI: } P<0.01 \\
\text { - VFI: } P<0.01\end{array}$ & \multicolumn{5}{|c|}{ None } \\
\hline \multirow{4}{*}{ [11] } & \multirow{4}{*}{$\begin{array}{l}-48 \text { PE }(8.4 \%) \\
\text { - } 10 \text { early PE }(1.7 \%)\end{array}$} & \multirow{4}{*}{$\begin{array}{c}\text { PE lower in women who } \\
\text { developed PE/ early PE } \\
\text { • VI: } P=0.14 \\
\text { • FI: } P=0.06 \\
\text { - VFI: } P=0.06\end{array}$} & \multirow[b]{2}{*}{ VI } & \multicolumn{2}{|r|}{$\mathrm{PE}$} & \multicolumn{2}{|c|}{ Early PE } \\
\hline & & & & \multicolumn{2}{|c|}{\begin{tabular}{c|} 
AUC 0.77 \\
$10 \%$ FPR: $45 \%$ senstivity \\
20\%FPR:55\%senstivity
\end{tabular}} & \multicolumn{2}{|c|}{$\begin{array}{c}\text { AUC } 0.89 \\
\text { 10\%FPR:45\%senstivity } \\
\text { 20\%FPR:55\%senstivity }\end{array}$} \\
\hline & & & FI & \multicolumn{2}{|c|}{\begin{tabular}{|c|} 
AUC 0.75 \\
10\%FPR:44\%senstivity \\
20\%FPR:56\%senstivity
\end{tabular}} & \multicolumn{2}{|c|}{$\begin{array}{c}\text { AUC } 0.89 \\
\text { 10\%FPR:79\%senstivity } \\
\text { 20\%FPR:79\%senstivity }\end{array}$} \\
\hline & & & VFI & \multicolumn{2}{|c|}{\begin{tabular}{|c|} 
AUC 0.77 \\
10\%FPR:44\%senstivity \\
20\%FPR:57\%senstivity
\end{tabular}} & \multicolumn{2}{|c|}{$\begin{array}{c}\text { AUC } 0.89 \\
\text { 10\%FPR:79\%senstivity } \\
\text { 20\%FPR:79\%senstivity }\end{array}$} \\
\hline \multirow{9}{*}{ [12] } & \multirow{9}{*}{$\begin{array}{l}\text { - } 44 \text { PE }(44 \%) \\
\text { - High risk: } 38 \text { PE }(76 \%) \\
\text { - Control: } 6 \text { PE (12\%) }\end{array}$} & \multirow{9}{*}{$\begin{array}{l}\text { VI and FI were lower in } \\
\text { the High-risk group } \\
\cdot \text { VI: } P<0.001 \\
\text { • FI: } P=0.263 \\
\text { - VFI: } P<0.001\end{array}$} & \multicolumn{2}{|c|}{ High risk } & VI (\%) & FI & VFI \\
\hline & & & \multicolumn{2}{|c|}{ Cutoff } & $<15.72$ & $<41.7$ & $<5.1$ \\
\hline & & & \multirow{2}{*}{\multicolumn{2}{|c|}{$\begin{array}{l}\text { Sensitivity } \\
\text { Specificity }\end{array}$}} & $76 \%$ & $80 \%$ & $80 \%$ \\
\hline & & & & & $84 \%$ & $82 \%$ & $92.1 \%$ \\
\hline & & & \multirow{2}{*}{\multicolumn{2}{|c|}{ Low risk }} & & & \\
\hline & & & & & VI (\%) & FI & VFI \\
\hline & & & \multicolumn{2}{|c|}{ Cutoff } & $<10.52$ & $<38.7$ & $<4.1$ \\
\hline & & & \multirow{2}{*}{\multicolumn{2}{|c|}{\begin{tabular}{|l} 
Sensitivity \\
Specificity \\
\end{tabular}}} & $66.2 \%$ & $70.7 \%$ & $70.6 \%$ \\
\hline & & & & & $94.7 \%$ & $95.8 \%$ & $86 \%$ \\
\hline [13] & - 10 PE (2.6\%) & $\begin{array}{c}\text { VI was lower in cases of PE } \\
\cdot \text { VI: } P=0.0007\end{array}$ & \multicolumn{5}{|c|}{$\begin{array}{l}\text { ROC curve for detection of PE using VI } \\
\text { (image presented, no numerical data) }\end{array}$} \\
\hline [14] & $\begin{array}{l}-66 \text { controls } \\
-62 \text { pt. with hypertensive } \\
\text { disorders }\end{array}$ & $\begin{array}{l}\text {-Placental volumes not reduced } \\
\text { in hypertensive group }(P>0.05) \\
\text {-VI and VFI were reduced in } \\
\text { hypertensive group }(P<0.01)\end{array}$ & & & & & \\
\hline [15] & $\begin{array}{l}\text { - } 126 \text { were normotensive } \\
\text { - } 38 \text { with gestational hypertension } \\
\text { - } 40 \text { with mild PE } \\
\text { - } 50 \text { with severe PE }\end{array}$ & $\begin{array}{l}\text {-SBP and DBP were higher } \\
\text { in hypertensive group } \\
\text {-VI and VFI were lower } \\
\text { in hypertensive group } \\
(P<0.01),(P=0.014) \\
\text {-FI not differ between groups }\end{array}$ & & & & & \\
\hline [16] & $\begin{array}{l}\text { - } 109 \text { were normotensive } \\
\text { - } 43 \text { with chronic hypertension } \\
\text { - } 57 \text { with gestational hypertension } \\
\text { - } 17 \text { with PE }\end{array}$ & $\begin{array}{l}\text { - placental volumes were } \\
\text { lower in all three pathological } \\
\text { groups }(P<0.001) \\
\text { - VI and VFI were lower } \\
\text { in hypertensive group } \\
(P<0.001),(P=0.003) \\
\text {-FI not differ between } \\
\text { groups }(P=0.141)\end{array}$ & & & & & \\
\hline
\end{tabular}

PE: Pre-eclampsia, VI: Vascularization index, FI: Flow index, VFI: Vascularization flow index, AUC: Area under curve, FPR: False positive rate, ROC: Receiver operating characteristic 


\section{CONCLUSION}

Pregnancies complicated by hypertensive disorders were associated with reduced placental vascularity but not reduced placental volumes.

\section{LIMITATIONS OF STUDY}

Vascularization indices should be conducted in the first trimester to determine whether this decreased vascularization was a causative effect or was consequential to hypertensive disorders in pregnancy.

\section{CONFLICT OF INTEREST}

There are no conflicts of interest.

\section{REFERENCES}

1. Abad C, Proverbio T and Pinero S. Preeclampsia, placenta, oxidative stress, and PMCA. Hypertense pregnancy; a HuGE review. American journal of epidemiology 2012;180(4): 335-45.

2. Hutcheon J, Lisonkova $\mathrm{S}$ and Joseph $\mathrm{K}$. Epidemiology of preeclampsia and the other hypertensive disorders of pregnancy. Best practice and research clinical obstetrics and gynecology 2011; (25): 391-403.

3. Neil O, Wright D, Syngelaki A, et al. Competing risks model in screening for preeclampsia by maternal factors and biomarkers at 11-13 weeks' gestation. American journal of obstetrics and gynecology 2016; (1): 103-11.

4. Schuchter K, Metzenbauer M, Hafner E, et al. Uterine artery Doppler and placental volume in the first trimester in the prediction of pregnancy complications. Ultrasound in obstetrics and gynecology 2013;18(6): 590-2.

5. Mihu C, Drugan T and Mihu D. Contribution of 3D power Doppler ultrasound to the evaluation of placental circulation in normal pregnancies and pregnancies complicated by preeclampsia. $\mathrm{J}$ Perinat Med 2012; 40: 359-64.

6. Brown $\mathrm{D}$, Dueker $\mathrm{N}$, Jamieson $\mathrm{D}$, et al. preeclampsia and the risk of ischemic stroke among young women. Results from the stroke prevention in young woman study. Stroke 2006; 37: 1055-9.

7. Cunningham $\mathrm{F}$, Gant $\mathrm{N}$, Leveno $\mathrm{K}$, et al. Hypertensive disorders in pregnancy. William obstetrics 24th ed. 2014, New York, McgrawHill; 761-808.
8. Paual C, Ruano R, Camppos J, et al. Quantitative analysis of placental vasculature by threedimensional power Doppler ultrasonography in normal pregnancies from 12 to 40 weeks gestation. Placenta 2009; 30: 142-8.

9. Plasencia W., Rutjes A., Westwood M., et al. First trimester placental volume and vascular indices in pregnancies complicated by preeclampsia, Prenat. Diagn 2015; (35): 1247-1254.

10. Demers S., Girard M., Roberge S., et al. First trimester placental, Myometrial Blood, Perfusion measured by three-dimensional power Doppler in preeclampsia, Amer J. Perinatol. 2015;(32): 920-926.

11. Hannaford K., Tuuli M., Goetzinger K., et al. First trimester three-dimensional power Doppler placental vascularization indices from the whole placenta versus the placental bed to predict preeclampsia. Does pregnancy-associated plasma protein a or uterine artery Doppler sonography help? J Ultrasound Med. 2015;(24): 965- 970.

12. Hashish N., Hassan A., El-Semary A., et al. Could 3D placental volume and perfusion indices measured at 1114- weeks predict occurrence of preeclampsia in high risk pregnant women? J. Matern. Neonatal Med. 2015;(28): 1094- 1098.

13. Hafner E., Metzenbauer M., Stumpflen I., et al. First trimester placental and myometrial blood perfusion measured by 3D power Doppler in normal and unfavorable outcome pregnancies, Placenta 2010;(31): 756- 763

14. Pimenta E., Silva de paula C., Fox A., et al. Threedimensional sonographic assessment of placental volume and vascularization in pregnancies complicated by hypertensive disorders. The American Institute of Ultrasound Medicine $\mathbf{J}$ Ultrasound Med 2014; 33(4): PP: 483-91.

15. Yan T., Zhang T. and Han Z. Placental Vascularization alteration in hypertensive disorders complicating pregnancy (HDCP) and small for gestational age with HDCP using threedimensional power Doppler in a prospective case control study. BMC pregnancy and childbirth 2015; $15: 240$

16. Suranyi A., Altorjay A., Kaiser L., et al. Evaluation of placental vascularization by threedimensional ultrasound examination in second and third trimester of pregnancies complicated by chronic hypertension, gestational hypertension or preeclampsia. An international journal of women's cardiovascular health 2017; PP 1-9. 